\title{
Réponses aux commentaires de Greiner et Burda
}

\author{
Markus Erismann
}

Psychotherapie-Wissenschaft 10 (2) 842020

www.psychotherapie-wissenschaft.info

CC BY-NC-ND

https://doi.org/10.30820/1664-9583-2020-2-84

Mots clés : « connaissance » et "perspicacité », différence de soi, identité, réflexion, conversation

Dans l'article suivant, je donne des réponses aux commentaires de Greiner et Burda publiés dans le dernier numéro (Psychotherapie-Wissenschaft, 9[2]).

Greiner (2019) différencie "Wissen in technisch-funktionaler Hinsicht (1. Konstitutionskriterium) » et " Erkenntnis in kritisch-reflexiver Hinsicht (2. Konstitutionskriterium) » en référence à Wallner. Pour constituer une science académique, la psychothérapie nécessite l'acquisition de «Erkenntnis », c'est-à-dire elle doit « sich darüber hinaus auch um die systematische Produktion vertiefter Einsichten in die komplexen Strukturen ibrer professionellen Aktivitäten bemühen ».

Selon Greiner, la « innerwissenschaftliche Vielschichtigkeits- und Fremdartigkeitsstruktur » et la " fachimmanente Reflexions-Provokation » forment un critère de délimitation et de qualité pour la psychothérapie. Cette caractérisation concise de la psychothérapie correspond à ma conception méthodologique de la psychothérapie et au deuxième critère constitutionnel de Greiner. Je voudrais donc contredire la critique de Greiner selon laquelle je n'ai fait référence qu'au premier critère constitutionnel.

Burda (2019) formule ses hésitations quant à mes attentes envers les psychothérapeutes d'avoir une conscience de soi prononcée de leur pensée et de leurs actions thérapeutiques. Il montre comment cette autoréflexivité concernant la théorie scientifique conduit à un cercle. Selon Burda, ce cercle peut être évité en prenant une perspective externe, une vue de l'extérieur, et en faisant une distinction claire entre la psychothérapie et la science de la psychothérapie. J'accepte les deux aspects.

Il y a deux raisons pour lesquelles je garde mes attentes d'un niveau élevé d'autoréflexivité en psychothérapie : 1. La réflexion joue un excellent rôle en psychothérapie. Greiner a justement décrit cela comme une provocation de la réflexion. 2. La science de la psychothérapie ne peut servir de médiateur entre la philosophie des sciences et les écoles de psychothérapie que si les psychothérapeutes individuels intègrent les connaissances de la science de la psychothérapie. Cela suppose la capacité de se remettre en question.

À mon avis, les psychothérapeutes peuvent se distancer de leur travail et réfléchir sur la pratique psychothérapeutique et sa base théorique. Au moins temporairement, ils adoptent le point de vue de la science de la psychothé- rapie. À mon avis, cette réflexion n'a pas besoin d'être reliée à un certain contexte philosophique. Au contraire, je plaide pour la plus grande indépendance possible de cette activité réflexive.

Une note sur le concept de différence de soi : Selon Burda, la différence de soi est un principe général qui affecte toutes les formes d'identité. La pratique psychothérapeutique peut consister à reconstruire et à sécuriser l'identité personnelle. Cette identité est une dimension de conscience de soi intacte (Stern, 2007 ; Scharfetter, 1999). Je me demande comment le concept de soi, qui est plus une question de dissolution que de construction d'identité, peut être utilisé en psychothérapie.

Enfin, trois questions générales pouvant intéresser la science de la psychothérapie :

1. Choisir une école psychothérapeutique particulière implique de choisir une perspective particulière. La capacité de changer de perspective semble être une condition préalable au dialogue entre les différentes écoles. Comment surmonter l'attachement à sa propre perspective?

2. Le changement de perspective nécessite la capacité de se remettre en question. Comment peut-on mieux comprendre les limites de sa propre perspective ? Cette compréhension est associée à l'humilité.

3. Comment formuler la relation entre la réflexion et le dialogue ? Comment conceptualiser la réflexion, comment structurer le dialogue pour acquérir des connaissances?

\section{L'Auteur}

Markus Erismann, docteur en philosophie, a étudié la philosophie et la langue et littérature allemandes à l'université de Zurich de 1983 à 1989 et a obtenu son doctorat en 1995/96 au département de philosophie de l'université de Zurich. Son travail se concentre sur l'épistémologie et la méthodologie.

\section{Contact}

Dr. Markus Erismann

Säntisstrasse 2

8008 Zürich

E-Mail : markus.erismann@bluewin.ch 